\title{
Broad-bandwidth Brillouin slow light in optical fibers
}

\author{
Miguel González Herráez*, Kwang-Yong Song ** and Luc Thévenaz \\ Nanophotonics and Metrology Lab. Ecole Polytechnique Federale de Lausanne, CH-1015, Switzerland \\ E-mail: miguelg@depeca.uah.es * On leave from Dept. of Electronics, Univ of Alcalá, Madrid, Spain. \\ ** Presently with the Department of Electrical Engineering, University of Tokyo, Japan.
}

\begin{abstract}
We experimentally demonstrate that Brillouin slow light with an arbitrary large bandwidth can be readily obtained in conventional optical fibers using a simple and inexpensive pump spectral broadening technique.

(C) 2006 Optical Society of America

OCIS codes: (999.9999) Slow light; (060.4370) Nonlinear optics, fibers; (290.5900) Scattering, Brillouin
\end{abstract}

\section{Introduction}

Recent experiments have demonstrated the possibility to achieve a wide group delay control in optical fibers by use of the stimulated Brillouin scattering (SBS) effect [1,2]. This technique is already thought to offer a vast potential for performing all-optical packet routing and random access memories [3]. In [4], it was experimentally demonstrated that arbitrarily large optically-controlled delays could be obtained by preventing pump depletion and amplified spontaneous Brillouin scattering. This simply requires the insertion of unidirectional broadband attenuators in the signal path, leaving the pump path lossless. In [5] an extremely wide group velocity control in the fiber was achieved using the same Brillouin principle. Group velocities as small as $71000 \mathrm{~km} / \mathrm{s}$, superluminal and even negative group velocities were observed. Up to now, however, the main limitation of this all-optical delaying technique has been its bandwidth, the natural Brillouin bandwidth being restricted to approximately $35 \mathrm{MHz}$ in conventional single-mode fibers. Thus, the temporal width of the optical pulses that can be delayed with this technique has so far been restricted to about $20 \mathrm{~ns}$. In this paper we demonstrate experimentally a method to overcome this limitation, thus opening the possibility to exploit slow light for the development of high-speed alloptical routers. This is the first demonstration of the control of both the delay and the bandwidth of slow-light. Our procedure is simple, inexpensive, uses off-the-shelf materials, and ensures that the bandwidth of the slow light can be matched to that of the signal. We believe that these results offer a basis for a technological application of this effect.

\section{Theory}

The process of SBS is usually described as the interaction of two counterpropagating waves, a strong pump wave and a weak probe wave. If a particular phase matching condition is satisfied (namely $f_{\text {pump }}=f_{\text {probe }}+v_{B}, v_{B}$ being the Brillouin shift), an acoustic wave is generated which scatters photons from the pump to the probe wave, stimulating the process. SBS can be regarded as a narrowband amplification process, in which a strong pump wave produces a narrowband gain in a spectral region around $f_{\text {pump }}-v_{B}$ and a loss around $f_{\text {pump }}+v_{B}$. According to the Kramers-Kronig relations, a refractive index change is associated with the Brillouin gain/loss process and a substantial change of the group index $n_{g}=n+\omega d n / d \omega$ follows as a result of the sharp index transition. When a perfectly coherent pump is used in the stimulated Brillouin interaction, the gain window appearing in the fiber transmission spectrum has a Lorentzian shape whose characteristic spectral width is around $35 \mathrm{MHz}$ in conventional single-mode fibers pumped at $1.55 \mu \mathrm{m}$. However, when the pump is modulated the gain bandwidth is given by the convolution of the pump spectrum and the Brillouin gain curve. Hence the effective Brillouin gain spectrum $g(\Delta v)$ is given by [6]:

$$
g(\Delta v)=P(\Delta v) \otimes g_{B}(\Delta v)
$$

where $\otimes$ denotes convolution, $P(\Delta v)$ is the normalized pump power spectral density (so that its integral is unity) and $g_{B}(\Delta v)$ is the characteristic Lorentzian gain of the Brillouin amplification process:

$$
g_{B}(\Delta v)=g_{B} \frac{1}{1-2 j\left(\Delta v / \Delta v_{B}\right)}
$$




\section{OTuA2.pdf}

for which $g_{B}$ is the linear Brillouin gain coefficient and $\Delta v_{B}$ is the characteristic Brillouin width. Hence an adequate pump modulation can be used to broaden at will the Brillouin interaction. A particularly useful case arises if the pump spectrum can also be approximated by a Lorentzian. In such conditions, the effective Brillouin gain shape remains Lorentzian, but shows a width equal to the sum of the characteristic Brillouin gain width and the pump spectral width. In this particular case, the delay obtained is given by $\Delta \tau=G /\left(\Delta v_{B}+\Delta v_{P}\right)$, where $G$ is the logarithmic gain suffered by the signal and $\Delta v_{P}$ is the pump spectral width. With no pump broadening $\left(\Delta v_{P}=0\right)$ the delay obtained amounts to $1 \mathrm{~ns}$ per $\mathrm{dB}$ gain [1]. Thus, for the same amount of signal gain, a tenfold increase of the bandwidth of the interaction comes at the expense of a tenfold reduction of the achieved delay. Since $G=g_{B} I_{P} L_{e f f} \Delta v_{B}\left(\Delta v_{B}+\Delta v_{P}\right)$, achieving the same absolute delay with a tenfold increase in the bandwidth of the interaction requires a 100-fold increase in the power of the pump or the effective length. More importantly, however, in terms of fractional delay (i.e. the delay divided by the pulse length) the same fractional delay with a tenfold increase in the bandwidth only requires a tenfold increase of the pump power.

\section{Experimental results}

Figure 1(a) depicts the experimental setup. Two conventional temperature and current-controlled distributed feedback (DFB) lasers are used to generate the pump and the probe, respectively. The frequency difference between the pump and probe lasers is set to the Brillouin shift of the fiber by adjusting the temperature and current settings of the lasers. To broaden the pump, a strongly attenuated pseudorandom binary sequence (PRBS) with a bit rate of $38 \mathrm{Mbit} / \mathrm{sec}$ is used to directly modulate the pump laser, yielding a smooth bell-shaped broadening of the pump spectrum. The broadened pump is amplified with an erbium-doped fiber amplifier (EDFA) and its intensity is controlled with a variable optical attenuator (VOA). The spectrum of the pump is monitored by the use of a fast detector connected to an electrical spectrum analyzer (ESA). The probe laser is modulated with an external electrooptic modulator to produce a $40-\mathrm{MHz}$ train of smooth 2.7 -ns wide pulses. A $6.7-\mathrm{km}$-long dispersion-shifted fiber with a Brillouin gain bandwidth of approximately $50 \mathrm{MHz}$ is used as the gain medium, in which the pump and probe are launched in opposite directions. The probe pulse train at the fiber output is amplified to a comfortable level using another EDFA, filtered and amplitude-controlled before being measured by another fast detector and a sampling oscilloscope. We tested our detection scheme for several probe powers with the pump turned off so as to ensure that there was no amplitude-dependent delaying measured by the detection scheme.

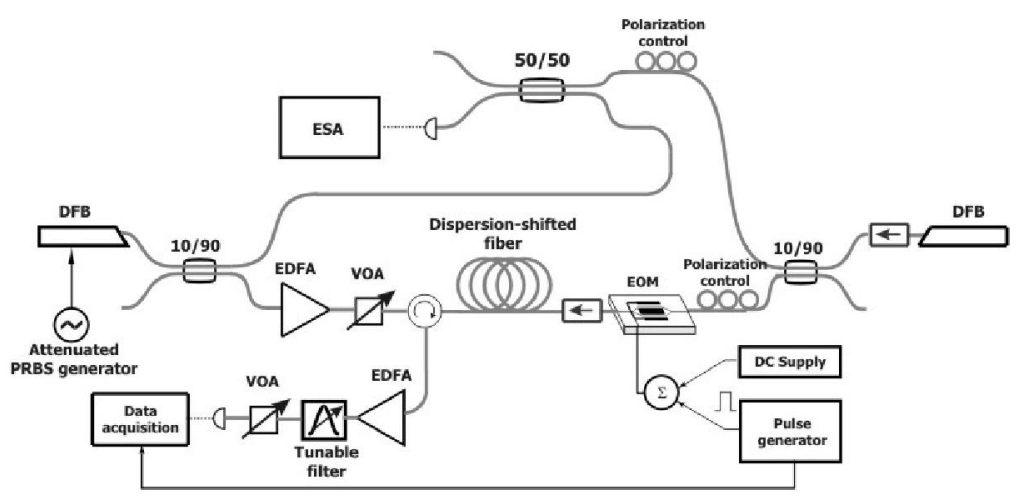

(a)

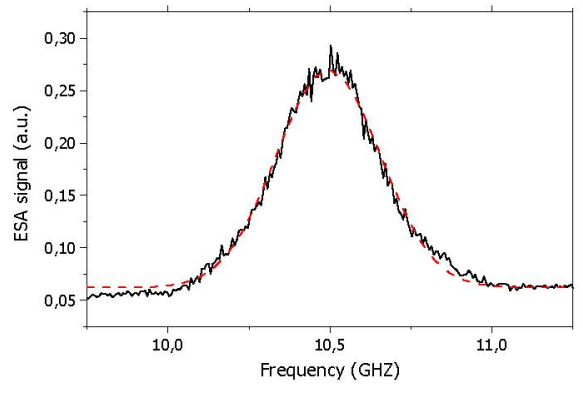

(b)

Fig. 1: (a) Experimental setup (b) pump-probe beating as recorded by the electrical spectrum analyzer.

Figure 1(b), shows the pump-probe beating as seen in a fast detector. We observe that the broadening is smooth and it can be well approximated by a Gaussian distribution. The pump broadening produced by this method is mainly due to phase modulation of the laser emission, the amplitude changes being nearly negligible. The relatively high bit rate of the PRBS generator ensures that the effective gain seen by the signal after propagation over the entire fiber length is effectively the convolution of the Brillouin gain of the fiber and the pump spectrum depicted in fig 1(b). The pump spectrum fits to a gaussian with a width of approximately $325 \mathrm{MHz}$. We evaluate the convolution of this gaussian with the lorentzian Brillouin gain and estimate that in this case the delay introduced per logarithmic gain corresponds to approximately $0.092 \mathrm{~ns} / \mathrm{dB}$, in good agreement with the analytical prediction made in the previous 


\section{OTuA2.pdf}

section. Figure 2(a) shows the delaying results of 2.7-ns pulses for several gain values, and figure 2(b) shows the achieved delay values as a function of the gain. We observe a linear dependence of the delay with the logarithmic gain with a slope of approximately $0.092 \mathrm{~ns} / \mathrm{dB}$, as expected from the theory. By tuning the probe frequency appropriately, we also performed measurements in the Brillouin loss region, obtaining pulse advancement with the same delay dependence. The achievable gain is limited to $30 \mathrm{~dB}$ due to the onset of spontaneous Brillouin scattering (the maximum achievable pump power in the fiber is approximately $30 \mathrm{~mW}$ ). Note that the threshold for amplified spontaneous Brillouin emission appears in this case for a power value that is roughly ten times bigger than in the non-broadened pump case. Although in this configuration the delay is limited to the tenth of the delay achieved in the non-broadened pump configuration, it must be pointed out that the maximum achievable fractional delay (i.e. the delay divided by the pulse length), which is the parameter of interest for real applications, is fully maintained ( $\approx 1.1$ ) and can be arbitrarily extended using the method described in [4]. A small residual broadening of the pulse is also observed for large gain values $(\approx 25 \%)$, as expected from the linear theory [4].

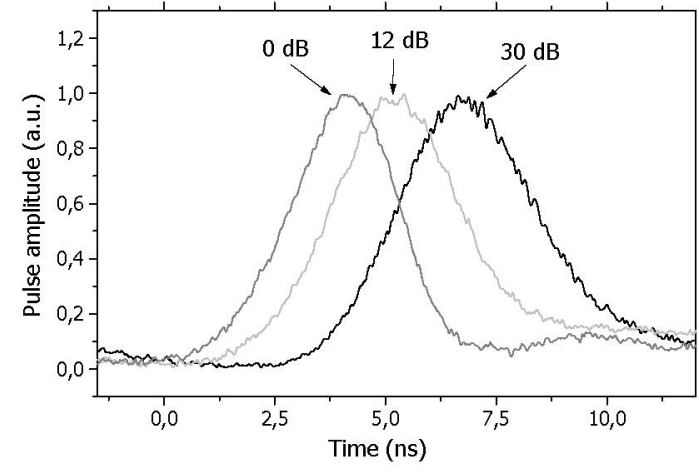

(a)

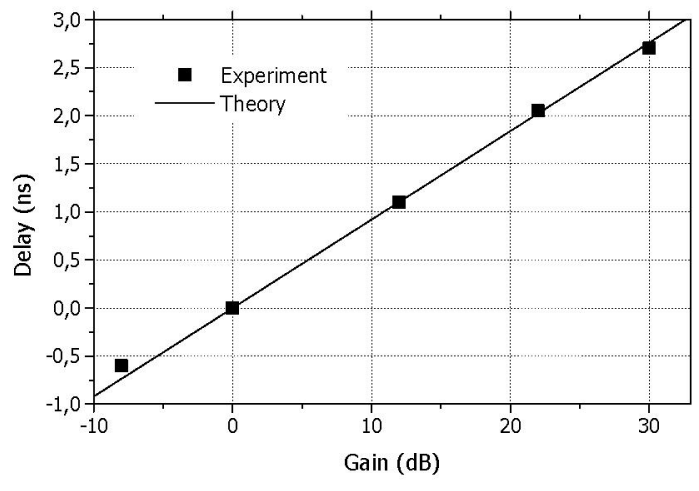

(b)

Fig. 2: (a) delaying results for three gain values (b) experimental and theoretical dependence of the delay as a function of the gain achieved in the fiber.

\section{Conclusion}

We have demonstrated experimentally a method for performing all-optical delaying and advancement of optical pulses of arbitrary bandwidth using stimulated Brillouin scattering that is based on the broadening of the pump spectrum. We have demonstrated a tenfold increase in the available bandwidth of the interaction, and we see no restriction to push this limit beyond to match telecommunication data rates. Furthermore, we see no theoretical drawback to extend this idea to other slow light setups, like those based on population oscillations [7].

We acknowledge financial support from the Swiss National Science Foundation through project 200021-109773/1. MGH also acknowledges funding from the University of Alcalá through project UAH PI2005/076.

\section{References}

[1] K. Y. Song, M. G. Herráez, and L. Thévenaz, "Observation of pulse delaying and advancement in optical fibers using stimulated Brillouin scattering," Opt. Express 13, 82-88 (2005), http://www.opticsexpress.org/abstract.cfm?URI=OPEX-13-1-82.

[2] Y. Okawachi, M.S. Bigelow, J. E. Sharping, Z. Zhu, A. Schweinsberg, D.J. Gauthier, R.W. Boyd, and A.L. Gaeta, "Tunable All-Optical Delays via Brillouin Slow Light in an Optical Fiber", Phys. Rev. Lett. 94153902 (2005).

[3] J. T. Mok and B. J. Eggleton "Expect more delays" Nature 433 811-812 (2005)

[4] K. Y. Song, M. G. Herráez, and L. Thévenaz, "Long optically-controlled delays in optical fibers," Opt. Lett. 30, 1782 (2005)

[5] M. González-Herráez, K.Y. Song and L. Thévenaz, "Long optically-controlled delays in optical fibers," Appl. Phys. Lett. 87, 081113 (2005)

[6] M. Denariez and G. Bret "Investigation of Rayleigh wings and Brillouin-stimulated scattering in liquids" Phys. Rev. 171, 160 (1968)

[7] P. C. Ku, F. Sedgwick, C. J. Chang-Hasnain P. Palinginis, T. Li, H. L. Wang, S. W. Chang and S. L. Chuang "Slow light in semiconductor quantum wells" Optics Letters 29 2291-2293 (2005). 